\title{
Franz Jeglitsch: Leoben, Politik und Gefüge
}

\section{Robert Danzer}

Institut für Struktur- und Funktionskeramik, Montanuniversität Leoben, Leoben, Österreich

Online publiziert 4. April 2019

Franz Jeglitsch (Abb. 1) wurde 1973 zum Professor für "Metallographie und metallkundliche Arbeitssverfahren" an der Montanuniversität Leoben ernannt. Er blieb an dieser Universität bis zu seiner Emeritierung im Jahr 2002 als Professor an verschiedenen Lehrstühlen und war auch als Rektor tätig. Schwerpunkt seiner Arbeiten war die Metallographie, mit der er sich während seiner gesamten Berufszeit auf hohem Niveau auseinander gesetzt hat. Er war auch immer aktiv im Fachausschuß "Metallographie“ der DGM tätig, dessen Obmann er für mehrere Jahre war.

Der Autor traf Franz Jeglitsch erstmals 1979, als er am Institut für "Metallkunde und Werkstoffprüfung" eine PostDoc-Position übernahm. Er erlebte Franz Jeglitsch als korrekten Institutschef, als Unterstützer und Förderer der wissenschaftlichen Arbeiten, als Mentor bei der Entwicklung seiner wissenschaftlichen Karriere, schließlich als Professorenkollege an der Montanuniversität Leoben und zuletzt auch als guten Freund, mit dem er nach langen Sitzungen der Österreichischen Akademie der Wissenschaften in Wien so manches Glas Wein geleert hat.

Diese lange gemeinsame Zeit hat natürlich zu vielen gemeinsamen Erlebnissen geführt, von denen einiges im Vortrag berichtet werden wird. Sie hat es dem Autor aber auch ermöglicht besser zu verstehen, was Franz Jeglitsch - neben seiner Familie, die für ihn ein ganz wichtiger und fester Anker war - wirklich interessiert und angetrieben hat: Im Mittelpunkt seines beruflichen Interesses stand seine Liebe zur Montanuniversität Leoben, sein Bemühen um Qualität in Forschung und Lehre und sein Bestreben, die Rahmenbedingungen dafür in Leoben, der Steiermark und in Österreich so gut wie möglich zu gestalten. Neben seiner Tätigkeit als Universitätsprofessor galt seine große Leiden-

R. Danzer: „Franz Jeglitsch: Leoben, Politik und Gefüge” Praktische Metallographie Sonderband 52 (2018), 57-6

\section{R. Danzer $(\square)$}

Institut für Struktur- und Funktionskeramik,

Montanuniversität Leoben,

Peter-Tunner Straße 5,

8700 Leoben, Österreich

robert.danzer@unileoben.ac.at schaft somit auch der Forschungspolitik. Dazu einige kurze Anmerkungen.

In der Steiermark spielen Werkstoffe, d.h. die Gewinnung der Rohstoffe, ihre Verarbeitung und Anwendung, seit Jahrtausenden eine bedeutende Rolle. Es gibt in der Umgebung von Leoben viele bedeutende Funde aus der Bronzezeit. Eine Periode aus der frühen Eisenzeit - die Hallstattzeit - ist sogar nach einem Ort etwa $100 \mathrm{~km}$ nordwestlich von

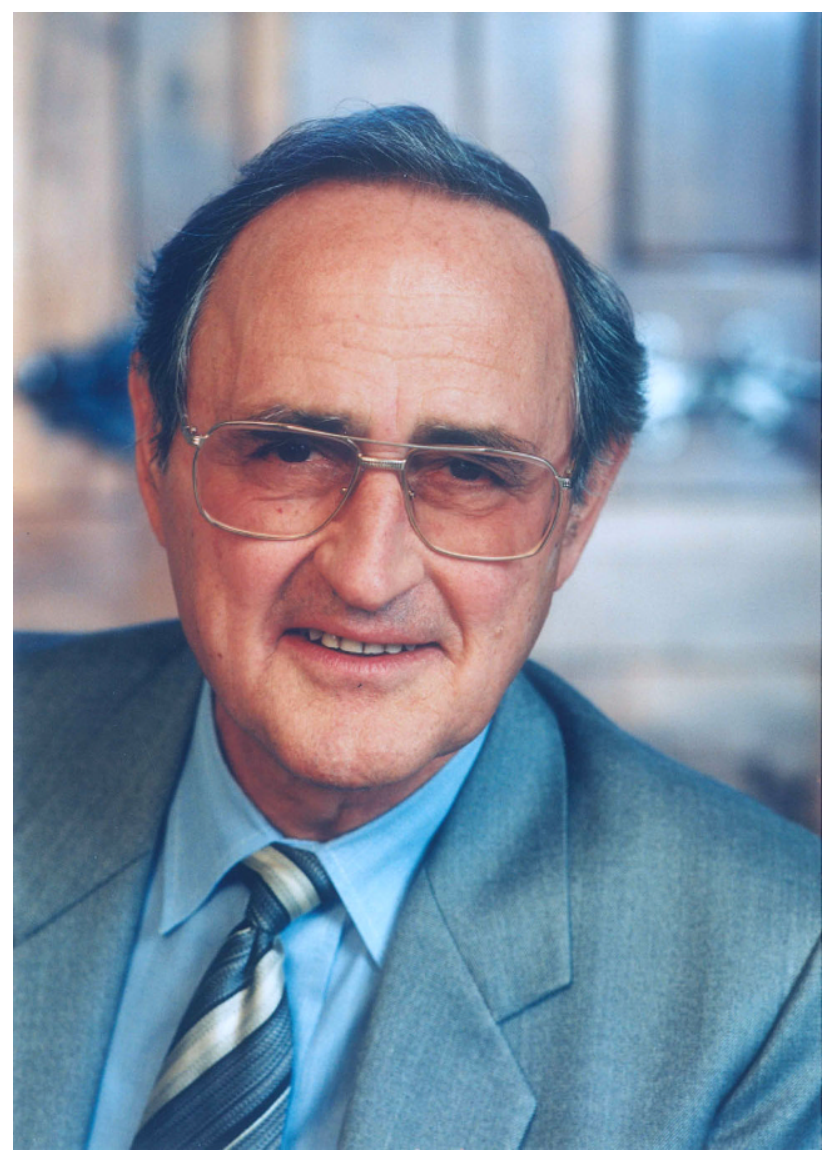

Abb. 1: o. Univ. Prof. Dr. Franz Jeglitsch 
Abb. 2: Das Ranking der Werkstoffstudien der Ecole Polytechnique de Lausanne aus dem Jahr 2012

\begin{abstract}
Einen grobartigen Erfolg konnte die Montanuniversita: außerdam im "Quantitative Ranking of Engineering Disciplines (QRED)" det School of Enginetring det Ecole polytechnigue federale de Lausanne (EPFL) erreichen: In Bereich det Wa:eriawissenschatien beieg:e de steitische Vorzengeunvets ta: it Oktober 2012 als zwecbeste turopaische Hochschule Rang 7. De Platze 1 bis 5 gingen an amerix anische Forschungstinnchtungen. gatolg: von der University of Cambridge. De Bewertung des einzeinen Hochschulen im QRED-Ranking basiert zu je 30 Prozent auf der Anzahl an wissenschaftichen Publokstionen (Anikel und Konferenzbesrajoe) pro Ptolessor sowe aut des Anzahl an Ztierungen pro Publikation in den zwe Jahren nach deren Veroffentichung.
\end{abstract}

\begin{tabular}{|c|c|c|c|c|c|c|}
\hline $\begin{array}{l}\text { World } \\
\text { Ronle }\end{array}$ & Institution & Countey & & $\begin{array}{l}\text { Pub// } \\
\text { Faculty }\end{array}$ & $\begin{array}{l}\text { Cltotionsy } \\
\text { Prib }\end{array}$ & $\begin{array}{l}\text { Q } \\
\text { Factor }\end{array}$ \\
\hline 1 & University of California Santa Barbara & U.S.A. & Is: & 86.6 & 98.8 & 100.0 \\
\hline 2 & Stanford University & U.S.A. & a: & 90.3 & 90.1 & 97.3 \\
\hline 3 & University of California Berkeley & U.S.A. & 는 & 79.8 & 100.0 & 97.0 \\
\hline 4 & Northwestern University (McCormick) & U.S.A. & $2=$ & 89.0 & 82.4 & 92.5 \\
\hline 5 & California Institute of Technology & U.S.A & 뇨다 & 92.6 & 76.5 & 91.2 \\
\hline 6 & University of Cambridoe & ark. & 喿 & $\sin 0$ & 46.6 & 201 \\
\hline 7 & Leoben & Austria & $=$ & 80.9 & 59.0 & 75.5 \\
\hline 8 & ETHZUnक (ETHZ) & Switzeriand & dाष्ट & 84.8 & 50.6 & 73.1 \\
\hline 9 & University of Illinois at Urbana.Champaign & U.S.A. & $\div$ & 56.9 & 73.0 & 70.1 \\
\hline 10 & EPF Lausanne (EPFL) & Switzeriand & 100 & 67.4 & 61.2 & 69.4 \\
\hline 11 & University of Erlangen-Nuremberg & Germany & $\Xi$ & 51.1 & 74.9 & 68.0 \\
\hline 12 & $\begin{array}{l}\text { Massachussetts Institute of Technology } \\
\text { (MII) }\end{array}$ & U.S.A. & $\div$ & 46.2 & 77.0 & 66.5 \\
\hline 13 & Imperial College & U.K. & 88 & 42.9 & 78.9 & 65.7 \\
\hline 14 & Cornell University & U.S.A. & 2 & 45.5 & 68.1 & 61.3 \\
\hline 15 & University of Michigan & U.S.A. & 28 & 48.8 & 59.9 & 58.6 \\
\hline
\end{tabular}

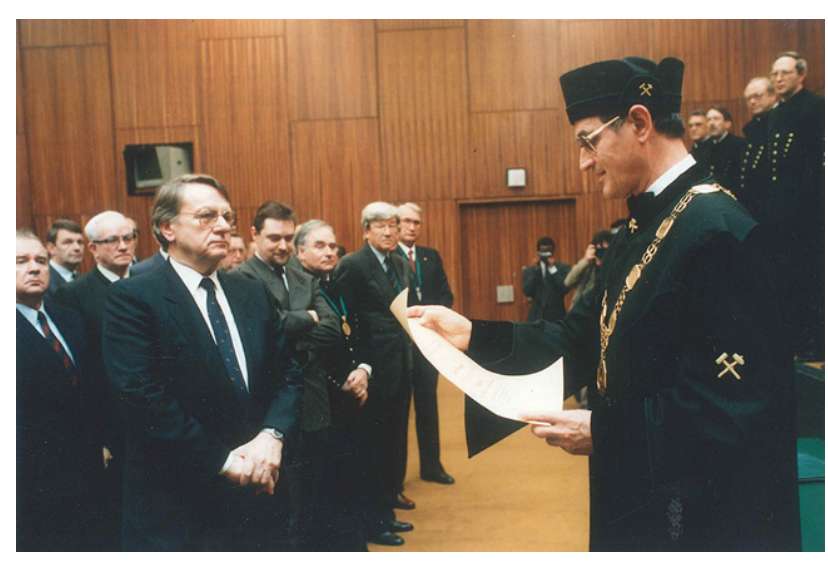

Abb. 3: Prof. G. Petzow wurde 1989 die Ehrendoktorwürde der Montanuniversität Leoben verliehen. Der Rektor, Prof. F. Jeglitsch, bei der Überreichung der Urkunde

Leoben benannt, das berühmte Ferrum Noricum kommt aus diesem Großraum. Am steirischen Erzberg, etwa $30 \mathrm{~km}$ nördlich von Leoben, wird seit mehr als 1000 Jahren Eisenerz abgebaut und in den umliegenden Tälern verhüttet. Der Abbau von Eisenerz und die Erzeugung von Eisen und Stahl waren vom Mittelalter bis heute für technische und wirtschaftliche Entwicklung unseres Landes von besonderer Bedeutung. Dabei spielte selbstverständlich die Verwendung von Stahl in der Waffentechnik eine große Rolle. Als im 19. Jahrhundert neue Produktionstechniken in England zum Einsatz kamen, welche die Konkurrenzfähigkeit der österreichischen Produkte bedrohten, gründete der Habsburgerprinz Erzherzog Johann 1840 eine Schule, in der Bergbau und die Erzeugung von Stahl unterrichtet wurden. Aus dieser Schule ging schließlich die Montanuniversität Leoben hervor.
Auch heute noch sind Werkstoffe und werkstoffnahe Technologien für Österreich von außergewöhnlicher Bedeutung. In Österreich sind Firmen mit Weltruf aus allen wichtigen Werkstoffbereichen beheimatet. Die Namen Voestalpine, Böhler, Planseewerke, Borealis, FACC, VeitschRadex, Epcos, Infineon oder Wienerberger mögen als wenige Beispiele dienen. Der Anteil dieser werkstoffnahen Industrien am Österreichischen Bruttonationalprodukt ist sehr hoch und sogar höher als der Anteil der Tourismusbranche. Dies ist der Hintergrund, auf dem die beruflichen Tätigkeiten von Franz Jeglitsch gründen.

Franz Jeglitsch hat in Leoben Metallurgie studiert. Er war als Assistent und Professor praktisch sein ganzes Berufsleben in Leoben tätig. Er hat die Bedeutung der Werkstoffe für Leoben und Österreich sehr früh verstanden und zielgerichtet auf den Ausbau Leobens als Zentrum der Werkstofforschung hingearbeitet. Er war bereits 1969 als junger Dozent gemeinsam mit dem damaligen Rektor G. Fettweis federführend an der Gründung der neuen Studienrichtung "Werkstoffwissenschaft" beteiligt und hat dabei wesentlich an der Entwicklung des Studienplans mitgewirkt. Das Studium ist im Dreiphasengebiet Grundlagen - Technologie Herstellung sehr nahe an der Grundlagenecke angesiedelt. Jeglitsch hatte großen Einfluß auf die Berufungen an der Universität. Es mag ein Zufall sein, daß die meisten Professoren aus den Grundlagenfächern an Themen aus dem Werkstoffbereich forschen. So forscht der Physikprofessor an Halbleitern, der Chemieprofessor an der lonenleitung in Oxidkeramiken und der Mechanikprofessor an der martensitischen Umwandlung von Stählen. Die Studienrichtung hatte im Schnitt der letzten Jahre etwa gleichauf mit der Studienrichtung "Petroleum Engineering“ die meisten Absolventen (Dipl.-Ing., Master). Die Zahl der Promotionen ist sogar mehr als doppelt so hoch wie bei allen anderen Leobener Studienrichtungen. 
Abb. 4: Tiefgeätztes Gefüge einer PZT-Keramik (a), geätzte Oberfläche einer Zerodur Glaskeramik (b), polierte Oberfläche eines Zn-Lotes (c), geätzte Oberfläche einer NTC-Keramik (d) Silbersulfid-Kristalle auf einem $\mathrm{LiNbO}_{3}$ Einkristall (e) und geätzte Oberfläche einer ZnO-Keramik (f). Der Autor bedankt sich bei Prof. Dr. Supancic (a), Prof. Dr. Lube (b), Dipl.-Ing. I. Kraleva (c), Dr. W. Harrer (d), Dr. M. Gruber (e) und Dr. N. Raidl (f) für das zur Verfügung stellen der Bilder
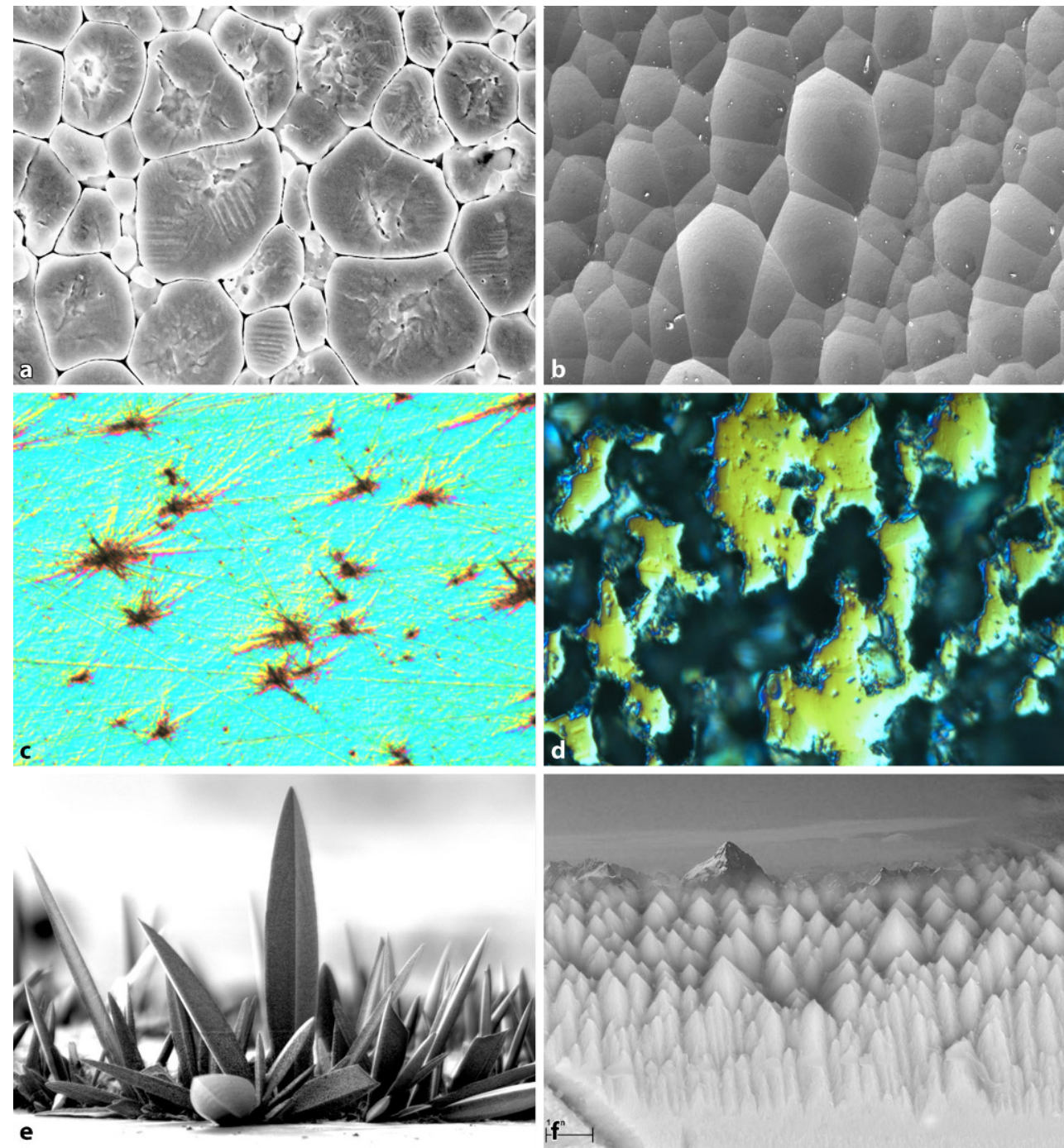

Jeglitsch "leitete" die Studienrichtung über viele Jahre und bis zu seiner Emeritierung. Hier hat er auf höchste Qualität in Forschung und Lehre geachtet, was er - im Sinne der universitären Grundidee - durch eine möglichst enge Verzahnung der Lehre mit der Forschung erzielen wollte. Diese Idee führte zu besonders vielen Forschungsprojekten, die teilweise durch den grundlagenorientierten Wissenschaftsfonds, in vielen Fällen aber auch durch die Industrie finanziert wurden. Das Publizieren - das in den Siebzigerjahren an einer Montanlehranstalt noch keine große Bedeutung hatte - wurde zu einem bedeutenden Faktor des wissenschaftlichen Disputs und der Qualitätskontrolle. Bei einer Erhebung aus dem Vorjahr hat sich ergeben, daß von Forschern der Studienrichtung Werkstoffwissenschaft, die etwa $10 \%$ der Lehrstühle der Universität aufweist, $73 \%$ aller "highly cited papers" (das sind Arbeiten mit mehr als 100 Zitierungen im SCl) der Universität verfaßt wurden. Im weltweiten Ranking der Werkstoffstudien, das von Ecole Polytechnique de Lausanne verfaßt wurde (Abb. 2), liegt Leoben als zweitbestes europäisches Studium an siebenter Stelle, nach Cambridge und noch vor der ETH Zürich. Nun sollte man solche Rankings nicht überbewerten, es zeigt aber, daß Jeglitsch sein Ziel, ein Studium hoher Qualität aufzubauen, erreicht hat.

Franz Jeglitsch hatte in Deutschland einen guten Freund, der in diesem Kreise ebenfalls sehr gut bekannt ist, nämlich Prof. Günter Petzow, mit dem er viele Ideen teilte (Abb. 3). Er folgte ihm auch in vielen Aufgaben und Funktionen, so beispielsweise als Vorsitzender des Arbeitskreises für Metallographie oder als Vorsitzender der DGM. Der Autor verdankt es vermutlich dieser Freundschaft, daß er von Petzow ein attraktives Angebot bekam, in Stuttgart am PML zu arbeiten. Dort lernte der Autor die deutsche Förderlandschaft kennen, die damals deutlich vielseitiger als die österreichische war. Seine Stelle wurde über ein Projekt des Materialforschungsprogramms des BMBF gefördert, das zur Hälfte von der Industrie und zur Hälfte von der öffentlichen Hand finanziert wurde. In Österreich gab es damals Förderprogramme, bei denen Grundlagenforschung zur Gänze, industrielle Forschung an Universitäten aber kaum gefördert wurde. Die österreichischen Forscher unseres Fachgebietes waren durch diese Politik gegenüber den deutschen Kollegen stark benachteiligt. In vielen Diskussionen, die der Autor mit Franz Jeglitsch führte, sprach dieser von der „För- 
derlücke", die es zu schließen galt. Die Forschungsquote betrug Ende der 80er-Jahre in Österreich etwa 1,3\%, und Jeglitsch dachte darüber nach, wie sie auf $2 \%$ gesteigert werden könnte. Damals begann Jeglitsch, sich in der Forschungspolitik zu engagieren und er war so erfolgreich, daß er etwas später auch (parteiloser) Abgeordneter im Steirischen Landtag wurde (von 1991-2000). Er war zuständig für die Bereiche Wissenschaft und Industrie sowie Kultur. Heute beträgt die Forschungsquote in Österreich 3,2\%.

Einer der wichtigsten Initiativen von Franz Jeglitsch war sein Engagement bei der Gründung der Christian Doppler Forschungsgesellschaft (gegründet 1988), in der das Deutsche Fördermodell weiterentwickelt wird. Begabte Forscher können nach bewilligter Antragstellung ein CD-Labor einrichten, das zur Hälfte von der interessierten Industrie und zur Hälfte von der öffentlichen Hand finanziert wird. Die CD-Gesellschaft ist heute in Österreich wahrscheinlich die am wenigsten bürokratische Fördermöglichkeit für Forschung im Nahtbereich Grundlagen und Anwendung. Ein CD-Labor wird für sieben Jahre eingerichtet und es können etwa fünf Wissenschaftler beschäftigt werden. 2017 gab es in Österreich 87 CD-Labore. Jeglitsch engagierte sich auch stark in der Forschungsgesellschaft Joanneum, der größten außeruniversitären Forschungsgesellschaft in
Österreich. Als 2004 in Deutschland die Exzellenzinitiative des BMBF gestartet wurde, kämpfte Jeglitsch in Österreich für ein ähnliches Programm. 2006 wurde auch in Österreich ein Exzellenzprogramm gestartet, und Franz Jeglitsch war der Spiritus Rektor für den Leobener Antrag für ein Exzellenzzentrum, das 2008 in der ersten Tranche des Programms bewilligt wurde. Das Materials Center Leoben (MCL) beschäftigt heute etwa 150 Wissenschaftler. Es gibt inzwischen zwei weitere Kompetenzzentren des Exzellenzprogrammes in Leoben (für Polymere und für Stahl). Die Steiermark erreichte im Jahr 2017 eine Forschungsquote von $5,2 \%$ und wurde damit - noch vor Baden Württemberg - zur forschungsstärksten Region Europas. Heute arbeiten in Leoben, einer Stadt mit etwa 25.000 Einwohnern, mehr als 500 Werkstoffwissenschaftler. Dies ist in hohem Maß auf das Wirken von Franz Jeglitsch zurückzuführen.

Als Forscher war Franz Jeglitsch vor allem an den Gefügen der Werkstoffe und ihrer Darstellung interessiert. Er zeigte in seinen Vorträgen viele Aufnahmen von Gefügen. Des Öfteren machte er den Scherz, unter diese auch von Künstlern gestaltete Bilder zu mischen. Er freute sich riesig, wenn keiner der Zuseher erkannte, was Natur war und was Kunst. So will auch der Autor seinen Vortrag mit einigen Bildern aus seinen Arbeiten beenden (Abb. 4). 